\title{
Methods for assessing the extent of exposure and effects of air pollution
}

\author{
Michal Krzyzanowski
}

\begin{abstract}
Background and objectives-In many places in Europe, the ambient air pollution exceeds the levels considered to be safe for health. The objective of the paper is to review and summarise the methods of assessment of its impact on health, and to indicate the contributions of various research disciplines, particularly environmental epidemiology.

Methods-The framework for assessment of impact is based on a four stage model: assessment of release of pollutant; assessment of exposure; assessment of the consequence; and risk estimation.

Results-Epidemiology is crucial in providing the data for the assessment of consequence. The criteria that determine the use of epidemiological studies for this task include lack of bias, correct control of confounding, and measured estimates of exposure. At present, those criteria are easier to satisfy for studies of short term effects on health than for the delayed consequences of exposure, or exposure accumulated over a prolonged period. Combinations of results from various populations through meta-analysis of existing studies or conducting multicentre studies is often necessary to increase the reliability of the consequence assessment stage.
\end{abstract}

Conclusion-To assess the impact on health systematically helps to focus on actions to limit air pollutants with the greatest impacts on human health and on method allows identification of the most pertinent questions which have to be answered by studies on relations between pollution and health and on exposure of populations to air pollutants. Epidemiology has considerable potential

WHO European

Centre for

(Occup Environ Med 1997;54:145-151)

Environment and

Health, Bilthoven, PO

Box 10, 3730 AA De

Bilt, The Netherlands

M Krzyzanowski

Correspondence to:

Dr Michal Krzyzanowski,

WHO European Centre for

Environment and Health

Bilthoven, PO Box 10, 3730

AA De Bilt, The

Netherlands.

Accepted 29 July 1996

Keywords: risk assessment; air pollution; environmen-

Health risks posed by air pollution are well recognised. Based on experience from episodes of high pollution, occupational health, and controlled human or animal exposure studies, individual countries have recomthe most affected populations. This to contribute to this research.

mended baseline controls on various pollutants in the atmosphere. The World Health Organisation (WHO) has recommended air quality guidelines. ${ }^{1}$ It was thought that "... inhalation of an air pollutant in concentrations and for exposure times below a guideline value will not have adverse effects on health ...". However, it has been admitted that "Compliance with recommendations regarding guideline values does not guarantee absolute exclusion of effects at levels below such values" because a section of the population may be exceptionally sensitive, and because of a "... combined exposure to various chemicals, or the exposure to the same chemical by various routes". Recent research on health effects of air pollution has indicated that adverse health effects in normal urban populations of several of the most common air pollutants can be found at concentrations close to, or below, the WHO guidelines. ${ }^{2}$ Based on this new data, the WHO has initiated an update of the guidelines with the aim of producing the revised assessment by the end of $1996 .^{3}$

Ambient concentrations of several air pollutants, such as $\mathrm{SO}_{2}$, suspended particulate matter, $\mathrm{NO}_{2}$, and $\mathrm{O}_{3}$ regularly exceed the recommended air quality guideline concentrations in many places in Europe. ${ }^{4}$ This causes concern about the impacts of the pollution on the health of the population. The relevant question is not "Does the impact exist?" but "What is its magnitude, what proportion of the population is affected, what is the severity of the health effects in individual subjects, and what is the public health significance of the exposure consequences?". These questions are asked by the general public and by the public health services. Often, they are also asked by representatives of various economic sectors that cause the air pollution..$^{5}$ Estimated impact on health is compared with the costs to the society related to the measures to be taken to reduce air pollution. This comparison should support management of the risk. When risk elimination is not realistic, risk reduction to an acceptable level can be proposed.

The ubjective of this paper is to summarise tal epidemiology management. As explained later, the impact assessment uses the existing data from research and from routine monitoring; it is, therefore, an interpretation of the scientific data, and not research by itself. At the same 
time, the framework of the assessment identifies topics which require more, or more focused, study. Some elements of the methodology have been applied in the preparation of the WHO-ECEH (European Centre for Environment and Health) report "Concern for Europe's tomorrow". ${ }^{4}$

\section{Framework for assessment of the health risk of air pollution}

The methods of assessment of the health impact can be presented with the framework of risk assessment adapted from earlier approaches by Covello and Merkhofer. ${ }^{6}$ Four main stages are: $(a)$ assessment of release of pollutants, (b) assessment of exposure, (c) assessment of the consequences, and $(d)$ risk estimation. All the stages are preceded by identification of the hazard.

In this paper, the discussion focuses on the non-cancer end points and the term impact assessment is used instead of risk assessment to distinguish two situations. The impact assessment considered here evaluates health effects of existing exposure. Provided the assumptions used in the assessment are valid, this should reflect impacts which have occurred in the population of concern. The risk assessment refers to the potential health impacts, assuming a hypothetical exposure scenario. In the analysis of components of air pollution with carcinogenic properties, risk assessment has been used; this reflects the assessment of the probability of the health outcome with a (long) latency period. ${ }^{\text {? }}$

With the multitude of substances with hazardous properties which pollute ambient and indoor air, the first step of impact assessment is to select the pollutants to be considered. These can be individual chemicals-such as $\mathrm{SO}_{2}, \mathrm{~Pb}$, or $\mathrm{O}_{3}$,-or mixtures-such as suspended particulate matter or environmental tobacco smoke. Sometimes an individual substance is considered to be an indicator representing a more complex mixture. ${ }^{8}$ The selection is often decided by the availability of data-both on the health effects of the pollutant and on its concentrations in the environment. Usually, the list of pollutants reduces to a few routinely monitored hazards or to substances for which good validated models are available. In investigations of specific pollution situations - for example, in the neighbourhood of a source of hazardous pollutants - the selection can be more specific because of the source. Knowledge of the human activity or natural process that is the source of risk may be necessary to select the hazardous substances for assessment. For risk management, the knowledge of those processes is essential.

\section{ASSESSMENT OF RELEASE OF POLLUTANTS}

One of the features of the well known, so called, classic pollutants is their presence in most locations where fossil fuels are used for heating, cooking, energy production, or transport. Their release is proportional to the intensity of these activities, to the quality of fuel used, the technology of combustion, and the presence of the measures to control emis- sion-such as filters in the end of pipes. Besides the emission from local sources, transportation of the pollutant in the atmosphere also contributes to pollution.

The release of secondary air pollutants depends on the presence of the precursor pollutants and the conditions (processes) promoting the transformations. Ozone is a secondary pollutant formed in the atmosphere from $\mathrm{NO}_{2}$ through a photochemical reaction involving short wavelength light. Ozone is a highly reactive gas; its reactions with NO reduces its concentrations near the sources of NO. The presence of volatile organic compounds influences the production/scavenging ratio and increases the steady state concentration of $\mathrm{O}_{3}$. The presence of high concentration peaks, with normal concentrations of primary pollutant, is usually dependent on the weather conditions - such as high amounts of sunshine, and low wind velocity. This identifies geographical areas where high peak concentrations of $\mathrm{O}_{3}$ can be expected.

\section{ASSESSMENT OF THE CONSEQUENCES}

At this stage, scientific information on the quantitative relation between the exposure level and extent of health impairment is summarised and its reliability is assessed. The relation can be expressed as a relative risk of a qualitative health outcome or a magnitude of change in a continuous health variable at a certain exposure level. Establishment of this relation is in the domain of toxicology and epidemiology.

Animal experiments have been the most common method to study the biological responses to chemicals. However, extrapolation of their results involves numerous uncertainties and assumptions. ${ }^{9}$ For the most common air pollutants, the evidence from human studies, both from the experiments with controlled exposure and from population studies, is crucial to assess their health consequences. Occupational studies or assessment of the health impact of episodes of high air pollution relate to unusual situations. Controlled human exposure studies are limited to observation of immediate health consequences of the exposures, and for ethical reasons cannot include people who are potentially the most susceptible to air pollution. Often, the few subjects included in the study limits the study power and precludes measurement of effects which vary between, and within, the subjects. Nevertheless, controlled studies of exposure to $\mathrm{O}_{3}$ in concentrations similar to those in ambient air have significantly contributed to the assessment of the relation of pulmonary function to the exposure. ${ }^{10}$

The study of the relation between health and low level air pollution is in the domain of epidemiological studies, investigating large populations to establish (sometimes weak) associations. ${ }^{11}$ However, the integrity of the results of the various methods used is important in indicating of the validity of the estimation of the relation between exposures and their consequences. 
For studies designed to establish causality and measure the health effect of the factor it is advisable to find and measure a pollutant (or its metabolite) close to the target organ. ${ }^{12}$ However, this requires invasive methods and is rarely possible in a human population study. Therefore, most studies designed to estimate of the association between the pollutant and health have used environmental indicators of contact of the study subjects with the pollutant assuming that there is a fairly good correlation between those indicators and the causative factor. ${ }^{13}$ Ambient concentrations of the pollutants have often been used as such indicators due to the availability of routinely collected air monitoring data. A potential drawback of this type of data results from the variability of concentrations of pollutants in time and space. Validity of exposure estimates based on ambient air monitoring should, therefore, be thoroughly evaluated in each epidemiological study. ${ }^{1415}$ Several pollutants, however, have been assessed for effects, and even measured without full understanding of the biological mechanisms leading to the health damage. This is the case for the important air pollutant, particulate matter. ${ }^{16}$

Exposure indicators must be validated so that the effects of the pollutant can be properly measured. Errors in the measurement of exposure, or misclassification of exposure status, may distort the real association and lead to false conclusions. When the errors in the measurement of exposure are similar for study subjects with various health outcomes (nondifferential misclassification), the error usually leads to underestimation of the true association between the pollution and outcome. ${ }^{12}$ Greater variability in measured than in true exposure leads to a smaller range in the measured exposure, and consequently to a smaller relative measure of effect-such as relative risk or odds ratio. The statistical power of such studies is also reduced, and it may be necessary to increase the sample size to achieve sufficient power. ${ }^{17}$ However, in some situations non-differential misclassification of exposure may result in an increase in the apparent risk estimate. One example of this occurs when the error in exposure estimate depends on the true exposure-for example, is greater with low pollution than with high pollution. ${ }^{18}$ When the bias in exposure measurement is different in groups with different health-for example, when the exposure is overestimated more often in sick than in healthy subjects-the results of the study may both underestimate or overestimate the true relation. Such errors, therefore, make the study unusable for assessment of health risk.

Errors in measurement of confounding variables may also bias results of the study, and the adjustment with a poor measure of the confounder may be equivalent to no control for confounding. The bias may cause the association to seem stronger or weaker than in reality. ${ }^{12}$

In summary, several criteria determine the use of an epidemiological study for quantitative risk assessment, and for assessment of its consequences to health in particular. ${ }^{19}$ Assuming that there is a positive association between the exposure and health variables, the study must be unbiased, confounding must be eliminated, and quantitative, and valid, exposure estimates must be available. Although, until recently, epidemiological studies have been criticised for not being able to cope with these criteria sufficiently well, there are a growing number of studies which can be, and are, used for impact assessment.

Most of those studies deal with health consequences of short term variability of air pollution levels. ${ }^{2}$ Temporal studies with aggregated data on mortality or hospital admissions assess health and exposure variables at the population level." The "all cause" mortality is considered in many studies as it is often available as the only indicator. The specificity of this indicator of health outcome is far from satisfactory. When the cause specific rates are considered, the disease which may be causally related to the increased air pollution may be mentioned as a contributing cause of death and so would not be available for analysis. ${ }^{20}$ Less severe health outcomes may be more appropriate for the studies of the effects of air pollution; reports from hospitals on admissions for respiratory diseases have been used for this purpose. ${ }^{21}$ The morbidity indicators based on health services may be related to the activity pattern of the services to a greater extent than to health of the population and this must be carefully considered in the analysis. ${ }^{22}$ The indicator of air pollution usually is an average concentration of the pollutant calculated from the data routinely collected in the area of residence of the studied population. ${ }^{23}$ This method certainly lacks precision and, in most cases, the analyses that use it will underestimate the effect of specific concentrations of a pollutant on health.

Panel studies consider information collected over a particular time (weeks or months) from a selected group of people. In comparison with the temporal studies of aggregated data, panel studies are more labour intensive and, therefore, less common. Specially collected information gives a more specific and more sensitive description of the health variables potentially related to air pollution, and provides potentially a better exposure indicator. ${ }^{24} \cdot 26$

In the temporal studies with aggregated data and in the panel studies, a health variable is correlated, after adjustment for confounding by a variety of time related factors, with the air pollution indicator relevant to the time unit. Recent developments of statistical techniques which are able to account for peculiarities of the data (autocorrelation, periodic changes) have contributed to measurement of these correlations. Considering types of errors in assessment of health and exposure variables, as well as the possible overadjustment for the time changing covariates, it can be argued that such studies provide a conservative estimate of the health consequences of the pollution.

Less common are the studies on health consequences of measured exposures that are pro- 
longed, and have a latency period. These studies have numerous technical difficulties such as the study duration (in cohort designs), problems with measurement of exposure over a prolonged period (or validity of retrospective exposure assessment), or appropriate control of confounding in studies comparing populations living in different environmental conditions. Nevertheless, such studies have been completed and have provided important indications for the long term health impacts of air pollution. ${ }^{27} 28$ Information on health consequences is usually better defined in the longitudinal studies than in temporal studies with aggregated data. Often, individual data on health and on potential confounders is available in such studies allowing for a better definition of the health end point, affected subgroups of the population, and possible individual characteristics correlated with the response to air pollution - for example, atopy.

Most of the common air pollutants are associated with health outcomes of multifactorial aetiology. Epidemiological studies aim to compare the frequency of those outcomes (or distribution of a continuous health variable) between groups exposed to different amounts of the studied pollutant. The desired outcome of the studies is a curve indicating the risk at each level of exposure (exposure-effect curve), and not only the estimated increase in risk between two exposure groups.

An important question pertinent to the studies of health effects of air pollutants at low concentrations is the shape of the exposureeffect curve, and in particular, the existence of a concentration below which no adverse effects are found. It is a common task of toxicological analysis to find the "no observed adverse effect level (NOAEL)", used in assessment of health criteria for environmental factors. ${ }^{9}$ The assumption of the existence of such a safe level is a basis of threshold limit values (TLVs) established for occupational settings. For epidemiological studies of the general population, it is difficult to find the threshold level. It is mostly due to the uncertainty of the low (or no) exposure status in a general population study: even if the threshold exists, the (usually non-specific) health outcome can be found in the low exposure group. It may be impossible to find if the effect is related to the low level of pollution, to factors other than pollution, or to misclassification of the exposure. In studies of the most common air pollutants, it may not be possible to establish a non-exposed group as the whole population is, or was in the past, exposed to some concentration of the pollutants above zero. A further complication in finding the threshold is the different individual sensitivities. The threshold may exist but may be different in each person. The overall picture for the population may depend on the proportion of sensitive subjects in this population. Nevertheless, if an appropriate exposure range is measured with sufficient precision, epidemiological studies can attempt to assess the existence of the threshold. ${ }^{29}$

Results from individual studies may be sufficient if risk assessment relates to the popula- tion considered in the given study. Calculation of attributable proportion, together with the estimate of its confidence interval, may be included into the interpretation of study results. However, in most cases, the impact assessment is conducted in populations without the relevant data from epidemiological studies. The estimates of the strength of associations must be extrapolated from studies conducted elsewhere with the underlying assumption that the shape, and the magnitude, of the association remains unchanged in various populations. The validity of such an assumption should be supported by observations actually conducted in various populations and reviewed by individual experts or groups of experts. ${ }^{30-33}$

Formal review, and estimation of summary statistics reflecting the relation of selected and well specified health consequences of exposure based on several studies, are done in a metaanalysis. ${ }^{3435} \mathrm{~A}$ paper published recently summarises the main criteria to apply to this technique. ${ }^{36}$ Between the other relevant features, the ability of the meta-analysis to identify heterogeneity of effects among many studies should be emphasised. However, the divergence of methods, health end points, and exposure indicators used in various studies severely restrict present possibilities for metaanalysis in the epidemiology of air pollution. An important development of this is the organisation and implementation of multicentre studies, which by design allow comparison of associations between well defined and uniformly measured air pollution and health variables. ${ }^{23} 37$ Combined estimates of effect of air pollution based on such studies provide the best input to the assessment of the consequences to health.

\section{EXPOSURE ASSESSMENT}

The main elements considered when exposure information is used in impact assessment are:

- Representativeness of the available environmental data for the (exposure of) population at risk

- Averaging time appropriate for the link with health

- Correspondence of the exposure indicator with the indicator of the exposure-response (consequence) function used in the assessment.

An additional criterion, not necessary for the impact assessment but potentially useful for the recommendations related to the risk management after the assessment, is the ability to link the exposure with the source of pollution.

These points differ considerably from the aspects of exposure assessment recommended for epidemiological research studies mainly due to the feasibility of collection of the optimal data.

To assess whether the exposure information is representative of the population at risk requires that the monitoring stations of the air pollutant are located in residential areas and in other sites where substantial proportions of the population exposure to outdoor air pollution 
may occur. Stations located next to important sources of pollution or stations located far away from built up areas (impact stations or background stations, according to the European Commission terminology ${ }^{38}$ ) should not be (directly) used for health impact assessment. Based on the monitoring results, information on concentrations specific to time and space should be derived.

The simplest method to estimate the population exposure is based on the average value calculated from data measured at the stations operating in the region where the population lives-for example, the town. This one value (in one time unit) is then assigned to the entire population of concern. Such a method may be acceptable and has been used in several epidemiological studies of relations between the source of exposure and the consequences. ${ }^{2123}$ Also, the town specific mean concentration may well reflect the mean concentration experienced by the residents moving within the town during the time unit. ${ }^{39}$ This approach has also been used to estimate exposure of urban populations in Europe for the WHO report Concern for Europe's tomorrow. ${ }^{4}$

More complex methods that use mapping of the pollutant concentrations often support the monitoring data with statistical or dispersion modelling. Modelling and mapping techniques are well developed to describe mean concentrations of pollutants in large rural areas. $^{40}$ Their application in densely populated urban areas is difficult due to the required scale and precision, but several studies have attempted this approach. ${ }^{41}$ The area specific estimates of concentrations are then superimposed-for example, with geographical information systems techniques - on the spatial distribution of the population to produce population distribution of exposure in a given time unit. Although that type of modelling may improve spatial definition of the pollution and can account for spatial differences in density of the resident population, it does not usually account for movement of the population within the area. However, if improved data, better than presently available, on patterns of activity were to be included, such modelling may significantly improve exposure assessment in the population where impact assessment is conducted. Such data will be necessary when sufficient numbers of studies that use personal exposure data are available for assessment of health response, and are included in the consequence assessment part of the risk assessment.

\section{IMPACT ESTIMATION}

This stage combines information from the preceding stages with the aim of measuring the impact, to indicate its uncertainty or certainty, and to indicate the elements influencing the precision of the estimates. Further, estimation of impact identifies factors which may influence the risk-such as special exposure conditions or susceptible groups.

Quantitative estimate of the impact can be based on the calculation of the attributable proportion, indicating the fraction of the health outcome which can be attributed to the exposure in a given population (provided that there is a causal association between the exposure and the health outcome). With the distribution of exposure of the population found in the exposure assessment stage, and the relation between exposure and consequence identified, the attributable proportion can be calculated with the formula:

$$
\mathrm{AP}=\frac{\Sigma\left\{[\mathrm{RR}(\mathrm{c})-1]^{\star} \mathrm{p}(\mathrm{c})\right\}}{\Sigma\left[\mathrm{RR}(\mathrm{c})^{\star} \mathrm{p}(\mathrm{c})\right]}
$$

where: $R R(c)=$ relative risk for the health outcome in category $c$ of exposure, and

$\mathrm{p}(\mathrm{c})=$ proportion of target population in category $c$ of exposure. Knowing (or, often, assuming) a certain underlying frequency of the outcome in the population, I, the rate (or number of cases per unit population) attributed to the exposure in the population can be calculated as:

$$
\mathrm{I}_{\mathrm{E}}=\mathrm{I} \star \mathrm{AP}
$$

For a population of a given size, this can be converted to the estimated number of cases attributed to the exposure.

When the response to the harmful exposure is expressed as a change in a continuous variable of health status - for example, lung function, body weight, intelligence quotient-the estimate of impact corresponds to the estimated mean change of the variable in the exposed population. Using the information on the variability of the response (from the assessment of the consequences), the range of the impact should be estimated as well-for example, as the 90 th or 95 th percentile of the response-to show the impact in the particularly sensitive people.

Each of the values used to derive this number is associated with an estimation error or other sources of uncertainty (related to the use of assumptions and expert judgement). Those uncertainties have to be considered at each stage of the estimation to produce lower and upper boundaries of the estimate as well as the best expected value. More elaborate approaches include modelling of the distribution of impact probability-for example, with Monte Carlo techniques. Input data for such procedures include information on the range of probabilities of the estimates of exposure and their consequences. ${ }^{6}$ The same procedures can also be used in sensitivity analysis to assess the most important elements in estimation of the precision of the impact-such as the relation between exposures and their consequences or the distribution of the exposure in the studied population.

An approach similar to that used in sensitivity analysis can be used to estimate changes in the impacts due to various scenarios of modification of exposure. This can increase the usefulness of assessment for risk management.

\section{Discussion}

Extrapolation and practical application of research involves simplification, generalisa- 
tion, and application of assumptions which may be difficult to test. This is often criticised by researchers as the tight quality standards are mixed with less stringent methods of inference. However, these applications are the main justification of the studies. As a matter of principle, the research on environmental health is oriented towards finding the factors affecting the health of populations and preventing adverse impacts on health. Obviously, not all populations can be studied. Therefore, it is necessary to generalise the results obtained in one population to other groups. The precision of this generalisation may be relatively low, but even this may be sufficient to identify local priorities and to find preventive actions. The approximate result based on impact assessment from the best available knowledge is always more systematic and clearer than a subjective judgement based on emotions or arbitrary assumptions. ${ }^{42}$

The procedure of risk assessment may indicate important gaps in knowledge and imposes quality criteria for further, focused research. Reduction of the scientific uncertainties pertaining to the risk assessment should be a common goal of the scientists and the decision makers. Improved data from epidemiological studies and from the studies describing exposure patterns in the populations are needed to refine risk assessment. Without the progress in research, the assessment of health benefits from the reduction or prevention of exposures to air pollution will remain uncertain..$^{43}$ Environmental epidemiology, as a discipline studying the associations of interest in the general population, can contribute most relevant data provided the studies are conducted to the desired standards. ${ }^{44}$

Particular caution in the interpretation of the impact assessment should be exercised when the analysis is based on "indicator pollutants", or on other exposure indicators which only indirectly correspond to the pollutant causing the health effect. The correlation of this indicator with the true level of the exposure in one population may not be repeated in another population. This problem shows a need for more relevant exposure monitoring in the assessment of health risks.

An important limitation of the present methodology is its reliance on the "one pollutant-one outcome" approach. To some extent, this approach is dictated by the scarcity of studies on health effects of a combination of pollutants characteristic of ambient exposures, and particularly on their interactions. Measurement of the components of mixtures responsible for the health effect is important to propose actions which may efficiently reduce the impact on health. ${ }^{45}$ However, it is possible that the same pollutant may have different impacts in the presence, or absence, of increased concentrations of other pollutants. ${ }^{46}$ Further, it is possible that a susceptible sector of the population responds to various types of air pollution in a similar way. In such cases reduction of one of the pollutants may result in a smaller than expected decrease in health problems as the effect of the other pollutants will remain. Therefore, in situations in which effects of individual pollutants are difficult to separate, a reduction of the pollution mixture, and of all its indicator components, should be advised. For a more precise description of the relation between air pollution and exposure, and for more specific preventive actions, better epidemiological studies and understanding of mechanisms of action of the relevant pollutants are necessary.

In conclusion, application of a systematic impact assessment helps to focus on preventive actions for air pollutants with the greatest impacts on human health and on the most affected populations. Use of this methodology enables identification of the most pertinent questions which have to be answered by studies on relations between pollution and health and on population exposure to air pollutants. Epidemiology has a great potential to contribute to this research.

Several of the issues presented in this paper have been discussed at the meetings of the Working Group "Risk Assessment" of the European Concerted Action "Air pollution Assessment" of the European Concerted Action "Air pollution paper were provided by $R$ van Leeuwen and A Kuchuk. The views presented are those of the author and not necessarily the views of the WHO.

1 World Health Organisation. Air quality guidelines for Europe.Copenhagen: WHO Regional Office for Europe 1987. (WHO Regional Publications, European Series No 23.)

2 Brunekreef B, Dockery DW, Krzyzanowski $M$ Epidemiologic studies on short-term effects of low levels of major ambient air pollution components. Environ Health Perspect 1995;103(suppl 2):3-13.

3 World Health Organisation. Update and revision of the air quality guidelines for Europe. Copenhagen: WHO Regional quality guidelines for Europe. Copenhagen: WHO Regional

4 World Health Organisation. Concern for Europe's tomorrow: health and the environment in the WHO European Region. WHO European Centre for Environment and Health. WHO-Euro. Stuttgart: Wiss Verl-Ges, 1995.

5 Edwards $R$. Industry denies dangers of particle pollution New Scientist 1995;2002:5.

6 Covello VT, Merkhofer MW. Risk assessment methods. Approaches for assessing health and environmental risks. New York: Plenum Press, 1993.

7 Hemminki K, Pershagen G. Cancer risk of air pollution: epidemiological evidence. Environ Health Perspect 1994;102(suppl 4):187-92.

8 Boström CE, Almén J, Steen B, Westerholm R. Human exposure to urban air pollution. Environ Health Perspect exposure to urban air poll

9 World Health Organisation/IPCS. Assessing human health risks of chemicals: derivation of guidance values for healthbased exposure limits. Geneva: World Health Organization, 1994. (Environmental Health Criteria 170.)

10 Horstman DH, Folinsbee LJ, Ives PJ, Abdul-Salaam S, McDonnell WF. Ozone concentrations and pulmonary response relationships for 6.6-hour exposures with five hours of moderate exercise to $0.08,0.10$ and $0.12 \mathrm{ppm}$ Am Rev Respir Dis 1990;142:1158-63.

11 Katsouyanni K, ed. ECA Air Pollution Epidemiology Report Series. Study designs. Luxembourg: CEC DG XII, 1993. (Report No 4. EUR 15095 EN.)

12 Armstrong BK, White E, Saracci R Principles of exposure assessment in epidemiology. Oxford: Oxford University Press, 1994.

13 Lebret E. Models of human exposure based on environmental monitoring. Sci Total Environ 1995;168:179-86.

14 Seifert B. Validity criteria for exposure assessment methods. Sci Total Environ 1995;168:101-7.

15 World Health Organisation European Centre for Environment and Health. Methodology for assessment of exposure to environmental factors in application to epidemiological studies. Sci Total Environ 1995;168: 93-100.

16 Seaton A, MacNee W, Donaldson K, Godden D. Particulate air pollution and acute health effects. Lance 1995;345:176-8.

17 McKeown-Eyssen GE, Tibshirani R. Implications of measurement error in exposure for the sample sizes of casecontrol studies. Am $\mathcal{F}$ Epidemiol 1994;139:415-21.

18 Wacholder $S$. When measurement errors correlate with truth: supprising effects of non-diferrential misclassification. Epidemiology 1995;6:157-61

19 Hertz-Picciotto I. Epidemiology and quantitative risk assessment: a bridge from science to policy. Am f Public Health 1995;85:484-91. 
20 Schwartz J. What are people dying of on high air pollution days? Environ Res 1994;64:26-35.

21 Schwartz J. Air pollution and hospital admissions for respiratory disease. Epidemiology 1996;7:20-8.

22 Quenel P, Medina S, Pirard P, Momas I, Le Moullec Y, Festy B, Dab W. Health service based morbidity indicators as a measure of health effects of air pollution. Eur $\mathcal{F}$ Public Health 1994;4:201-6.

23 Katsouyanni K, Zmirou D, Spix C, Sunyer J, Schouten JP Pönkä A, et al. Short-term effects of air pollution on health: a European approach using epidemiological timehealth: a European approach using epidemiological timeseries data. The APHEA project: back
design. Eur Respir 7 1995;8:1030-8.

24 Hoek G, Brunekreef B. Effect of photochemical air pollution on acute respiratory symptoms in children. $A m \mathcal{F}$ Respir Crit Care Med 1995;151:27-32.

25 Krzyzanowski M, Quackenboss J, Lebowitz MD. Relation of peak respiratory flow rates and symptoms to ambien ozone. Arch Environ Health 1992;47:107-15

26 Quackenboss J, Krzyzanowski M, Lebowitz MD Exposure assessment approaches to evaluate respiratory health effects of particulate matter and nitrogen dioxide. $\mathcal{F}$ Expo Anal Environ Epidemiol 1991;1:83-107.

27 Abbey DE, Lebowitz MD, Mills PK, Petersen FF, Beeson WL, Burchette RJ. Long-term ambient concentrations of particulates and oxidants and development of chronic disease in a cohort of non-smoking California residents. disease in a cohort of non-sm
Inhal Toxicol 1995;7:19-34.

28 Dockery DW, Pope CA III, Xu X, Spengler JD, Ware JH Fay ME, et al. An association between air pollution and mortality in six US cities. $N$ Engl $f$ Med 1993;329. 1753-9.

29 Ulm K. A statistical method for assessing a threshold in epidemiological studies. Stat Med 1991;10:341-9.

30 Dockery DW, Pope CA III. Acute respiratory effects of particulate air pollution. Annu Rev Public Health 1994; 15:107-32.

31 Department of Health. Advisory Group on the Medical Aspects of Air Pollution Episodes. Third report: oxides of Aspects of Air Pollution Episodes.
nitrogen. London: HMSO, 1993.

32 Department of Health. Committee on the Medical Effects of Air Pollutants. Non-biological particles and health. of Air Pollutants. Non

33 World Health Organisation. Update and revision of the air quality guidelines for Europe. Meeting of the Working Group on Classical Air Pollutants. Copenhagen: WHO Regional Office for Europe, 1995. (EUR/ICP/EHAZ 94 05/PB01.) 34 Hasselblad V. Meta-analysis of environmental health data. Sci Total Environ 1994;160/161:545-58.

35 Schwartz J. Air pollution and daily mortality: a review and meta-analysis. Environ Res 1994;64:36-52.

36 Blair A, Burg J, Foran J, Gibb H, Greenland S, Morris R, et al. Guidelines for application of meta-analysis in environmental epidemiology. Regul Toxicol Pharmacol 1995;22:189-97.

37 Brunekreef B, Hoek G, Roemer W, Kalandidi A, Baldini G, Vondra V, et al. PEACE Study. (1) Background and design. Epidemiology 1995;6:S65.

38 European Commission for Europe (ECE). Conference of European Statistician. Readings in international environmental statistics.Geneva: United Nations, 1993. (United Nations Publication GE.93-32468.)

39 Pirard P, Quenel P, Lameloise P, Le Moullec Y. Etude de l'utilisation d'une moyenne arithmétique des mesures d'un réseau de surveillance comme indicateur de niveau de pollution atmosphérique en milieu urbain. Pollution Atmosphérique 1995;Avril-Juin:59-66.

40 Simpson D. Photochemical model calculations over Europe for two extended summer periods: 1985 and 1989. Model results and comparison with observations. 1989. Model results and comparison with

41 Briggs DJ, van der Veen AA, Pryl K, Collins S. Air pollution mapping in SAVIAH study. Epidemiology 1995;6: S32.

42 Goldstein $\mathrm{BD}$. The need to restore the public health base for environmental control. Am $\mathcal{F}$ Public Health 1995;85: 481-3.

43 Sexton $\mathrm{K}$. Science and policy in regulatory decision making: getting facts right about hazardous air pollutants. Environ Health Perspect 1995;102(suppl 6):213-22.

44 Shore RE. Epidemiologic data in risk assessment - imperfect but valuable [editorial]. Am $\mathcal{f}$ Public Health 1995; 85:474-5.

45 Dockery DW, Schwartz J. Particulate air pollution and mortality: more than Philadelphia story. Epidemiology mortality: more

46 Lebowitz MD, Quackenboss JJ, Krzyzanowski $M$ O'Rourke MK. Multipollutant exposures and health responses to particulate matter. Arch Environ Health 1992;47:71-5.

\section{Occupational and Environmental Medicine and the electronic age}

OEM has an Email address which is 100632.3615@compuserve.com.We welcome contact by Email, including letters to the editor. Some of our reviewers already send us their reports by Email, helping to speed up the peer review process.

We are moving towards electronic publishing and for some months now we have been asking authors to send us their revised papers on disk as well as a hard copy. I am delighted to report that nearly all our authors are managing to comply with this request. Oddly enough, the few authors who have not sent us a disk version of their revised papers have been almost exclusively from the United Kingdom. I would be interested in suggestions for why this might be. Perhaps United Kingdom based authors read our correspondence and instructions less assiduously? Watch for revised Instructions to Authors.

The Editor 